\title{
Spatial Variation in Infant Mortality with Geographically Weighted Poisson Regression (GWPR) Approach
}

\author{
Kristina Pestaria Sinaga, Manuntun Hutahaean², Petrus Gea ${ }^{3}$ \\ 1, 2, 3 University of Sumatera Utara, Faculty of Mathematics and Natural Science, Bioteknologi Road, Medan 20155, Indonesia
}

\begin{abstract}
Regression analysis is a statistical analysis that aims to model the relationship between response variable and predictor variable. If the response variable distributes Poisson, the regression model used is Poisson regression. The main problem of this method is if the method is applied to spatial data. To overcome the spatial data problem, the statistical method to be used is Geographically Weighted Poisson Regression (GWPR) i.e. the local form of Poisson regression where the location noted. The results showed that the GWPR model parameters estimation used Maximum Likelihood Estimator (MLE) and was solved by using Newton-Raphson iteration. In this study the concept of geographical circumstances was applied to Poisson regression. The GWPR model application of the data percentage of infant mortality in North Sumatra, Indonesia showed that by using different weighting, the variables affecting the number of infant mortality per district/city in North Sumatra were also different. Based on the value of the Akaike Information Criterion (AIC) between Poisson regression model and GWPR model, it was known that GWPR model with weighting function of bisquare kernel was the better model used to analyze the number of infant mortality in North Sumatra Province in 2013 because it had the smallest AIC value.
\end{abstract}

Keywords: Infant Mortality, Poisson Regression, GWPR

\section{Introduction}

By far the most common statistical modeling technique used in the social sciences is that of regression [5]. In standard application of regression, a dependent variable is linked to a set of independent variables with one of the main outputs of regression being the estimation of a parameter that links each independent variable to the dependent variable. A major problem with this technique when applied to spatial data often referred to as a global (spatial stationary) is that the processes being examined are assumed to be constant (stationary) over space - that is, one model fits all $[4,7,8]$.

However, a global regression model is only appropriate when it is reasonable to assume that the relationship between $\mathrm{Y}$ and $\mathrm{X}$ does not change across the study region. In some cases, there may be evidence to suggest that the relationship between the dependent and independent variables depends on the geographical location and that the parameters are not significant spatial variation that is not accounted for in the estimation of the global parameter estimates [4,5]. This variation is reffered to as spatial non-stationarity [1].

The Geographically Weighted Regression (GWR) approach is a statistical technique used to account for spatial non stationarity by celebrating a regression model which allows different relationships to exist at different location in space. Most applications of GWR have involved celebrating a model with a Gaussian error term (which is the geographically weighted equivalent of an OLS regression model). The concept of GWR can be applied to Generalized linear models (GLMs), including those based on the binomial or poisson distributions.

Poisson regression is used when the dependent variable refers to counts of the occurrences of some event over time or space and takes the form of any non - negative integer. The independent variables can be either qualitative, quantitative or a combination of both. In order to fit a geographically weighted poisson regression (GWPR) model, each observation must have information that describes its location. This information can be in the form of $(x, y)$ coordinates based on a two-dimensional Cartesian grid, or latitudelongitude coordinates which describe a poit on the Earth's surface.

The reminder of this paper is organized as follows. In the next section, we describe the theoretical framework of GWPR including how a GWPR model is calibrated. Afterward, the sampled Infant mortality data are reviewed for the application of GWPR methods. Finally, conclusions are summarized and future work is outlined.

\section{Generation of Data}

Infant mortality is death happened to children under one year old. Infant Mortality Rate (IMR) is the high possibility of infants dying before one year old, it is expressed in per thousand live births. Infant mortality is influenced by the health condition of housing and socio-economic circumstances of parents [3]. Socio-economic and cultural factors are determinants of infant morbidity and mortality, but these effects are indirect because these have to pass certain biological mechanisms which then will create morbidity risk, infant sickness, and if the infants do not cured, the infants will be deformed or dead. In this issue, infant morbidity and mortality are the problems.

The data used in this research were secondary data obtained from Central Bureau of Statistics i.e. the data of National 


\section{International Journal of Science and Research (IJSR) \\ ISSN (Online): 2319-7064}

Index Copernicus Value (2013): 6.14 | Impact Factor (2014): 5.611

Socio-Economic Survey (SUSENAS) in 2013 for North Sumatra Province, Indonesia [2]. To support the research process it was used computer program packages, namely software R 3.1.2 and GWR4.

The variables used were the percentage of infant mortality $(Y)$, percentage of deliveries performed with non-medical help $\left(X_{1}\right)$, average women's age at the first marriage $\left(X_{2}\right)$, average number of household expenditure per capita in a month $\left(X_{3}\right)$, average of exclusive breastfeeding $\left(X_{4}\right)$, Total health officers $\left(X_{5}\right)$, percentage of poor people $\left(X_{6}\right)$, Number of Health Facilities $\left(X_{7}\right)$, Latitude $\left(u_{i}\right)$, and longitude $\left(v_{i}\right)$.

\subsection{Poisson Regression Model}

Poisson regression is a form of regression analysis used to model data in the form of count (number), for example, the data are denoted by $\mathrm{Y}$ (the number of events occurred over a period of time and/or a particular region). Poisson regression assumes that random variable of $Y$ distributes Poisson. Random variable of $\mathrm{Y}$ is defined to have Poisson distribution if the density (odds function) is composed as follows [4] :

$$
f_{Y}(y)=f_{Y}(y ; \mu)=\left\{\begin{array}{cl}
\frac{e^{-\mu} \mu^{y}}{y !}, & y=0,1,2, \ldots \\
0 & \text { others }
\end{array}\right.
$$

With parameter $\mu>0$. Equation (1) is also called as Poisson likelihood function. Poisson regression model can be written as follows:

$$
\begin{array}{r}
\log \left(\mu_{i}\right)=\beta_{0}+\sum_{j=1}^{k} \beta_{j} x_{i j}, \quad i=1,2, \ldots, n \\
\text { With } \\
\mu_{i}=\mu_{i}\left(x_{i}\right)=\exp \left(\beta_{0}+\sum_{j=1}^{k} \beta_{j} x_{i j}\right)
\end{array}
$$

The Poisson regression parameter estimation is performed by using Maximum Likelihood Estimation (MLE). "The possibility function is as follows:

$$
L(y ; \beta)=\prod_{i=1}^{m} \frac{e^{-\exp \left(x_{i}^{T} \beta\right)} \exp \left(x_{i}^{T} \beta y_{i}\right)}{y_{i} !}
$$

The above equation will be assumed by using iterative technique producing maximum likelihood estimator for regression coefficient in $\hat{\beta}$

$$
\begin{aligned}
L(y ; \beta) & =\prod_{i=1}^{m} \frac{e^{-\exp \left(x_{i}^{T} \beta\right)} \exp \left(x_{i}^{\tau} \beta y_{i}\right)}{y_{i} !} \\
& =\sum_{i=1}^{m} \ln \left[\frac{e^{-\exp \left(x_{i}^{T} \beta\right)} \exp \left(x_{i}^{\tau} \beta y_{i}\right)}{y_{i} !}\right] \\
& =\sum_{i=1}^{m}\left[\ln \left(e^{-\exp \left(x_{i}^{T} \beta\right)}\right)+\ln \left(\exp \left(x_{i}^{\tau} \beta y_{i}\right)\right)-\ln y_{i} !\right] \\
& =\sum_{i=1}^{m}\left[x_{i}^{\tau} \beta y_{i}-\exp \left(x_{i}^{\tau} \beta\right)-\ln y_{i} !\right]
\end{aligned}
$$

To obtain its maximum likelihood value, it is used Newton Raphson iteration numerical calculation method because the equation is implicit.

\subsection{Geographically Weighted Regression (GWR)}

Geographically Weighted Regression (GWR) is a fairly recent contribution to modeling spatially heterogeneous processes [1]. The underlying idea of GWR is that parameters may be estimated anywhere in the study area given a dependent variable and a set of one or more independent variables which have been measured at places whose location is known. Taking Tobler's observation about nearness and similarity into account we might expect that if we wish to estimates parameters for a model at some location then observations which are nearer that location should have a greater weight in the estimation than observations which are further away [8].

GWR model can be formulated as below.

$$
\hat{y}_{i}=\beta_{0}\left(u_{i}, v_{i}\right)+\sum_{k=1}^{p} \beta_{k}\left(u_{i}, v_{i}\right) x_{i k}+\varepsilon_{i}
$$

where $\left\{\beta_{0}\left(u_{i}, v_{i}\right), \ldots, \beta_{k}\left(u_{i}, v_{i}\right)\right\}$ are $k+1$ continuous functions of the location (ui,vi) in the geographical study area. The $\varepsilon_{i}$ are random error terms [1].

\subsection{Geographically Weighted Poisson Regression (GWPR)}

Model GWPR is a local form of Poisson regression producing localized model parameter estimator for each point or location where the data are collected by assuming data distributing Poisson $\left(Y_{i} \sim\right.$ Poisson $\left.\left(\mu\left(x_{i}, \beta\right)\right)\right)$. Because response variable distributes Poisson, the likelihood function is as follows:

$$
I(\beta)=\prod_{i=1}^{n} \frac{\exp \left(-\mu\left(x_{i}, \beta\right)\right)\left(\mu\left(x_{i}, \beta\right)\right)^{y_{i}}}{y_{i} !}
$$

After likelihood form obtained, it is done natural logarithm operation so that equation (4) becomes:

$$
\operatorname{Ln} L(\beta)=\sum_{i=1}^{n}\left(-\mu\left(x_{i}, \beta\right)+y_{i} \operatorname{Ln} \mu\left(x_{i}, \beta\right)-\operatorname{Ln} y_{i} !\right)
$$

Based on equation (2), equation (5) can be written as follows:

$$
\operatorname{Ln} L(\beta)=\sum_{i=1}^{n} y_{i} x_{i}^{\tau} \beta-\sum_{i=1}^{n} \exp \left(x_{i}^{\tau}, \beta\right)-\sum_{i=1}^{n} \operatorname{Ln} y_{i} \text { ! }
$$

Geographical location factor is weighting factor of GWPR model. This factor has different value for each region showing local nature of GWPR model. Therefore weighting is given in the form of its log-likelihood for local GWPR model, so that it is obtained

$$
\operatorname{LnL}\left(\beta\left(u_{i} v_{i}\right)\right)=\sum_{i}^{\pi}\left(y_{i} x_{i}^{\tau} \beta\left(u_{i} v_{i}\right)-\exp \left(x_{i}^{\tau}, \beta\left(u_{i} v_{i}\right)\right)-\operatorname{Ln} y_{i} ! w_{t}\left(u_{i} v_{i}\right)\right.
$$

Parameter estimation is $\hat{\beta}\left(u_{i}, v_{i}\right)$ was obtained by the partial derivatives of above equation on $\beta\left(u_{j}, v_{j}\right)$ and its result

$$
\frac{\partial \operatorname{Ln} \mathrm{L}^{*}\left(\beta\left(u_{l} v_{l}\right)\right)}{\partial \beta^{\tau}\left(u_{j} v_{j}\right)}=\frac{\sum_{j=1}^{n}\left(y_{j} x_{j}^{\tau} \beta\left(u_{j} v_{j}\right)-\exp \left(x_{j}^{\tau}, \beta\left(u_{j} v_{j}\right)\right)-\operatorname{Ln} y_{l} ! w_{\nu}\left(u_{j} v_{j}\right)\right.}{\partial \beta^{r}\left(u_{j} v_{j}\right)}
$$




\section{International Journal of Science and Research (IJSR) \\ ISSN (Online): 2319-7064}

Index Copernicus Value (2013): 6.14 | Impact Factor (2014): 5.611

$$
=\sum_{i \alpha}^{n}\left(y_{i} x_{i}^{\tau} \beta\left(u_{i} v_{i}\right)-\exp \left(x_{i}^{\tau}, \beta\left(u_{i} v_{i}\right)\right)\right) w_{\psi}\left(u_{i} v_{i}\right)
$$

The estimated value is obtained by maximizing the differential form so that it is obtained:

$$
\frac{\partial \operatorname{Ln} \mathrm{L}^{*}\left(\beta\left(u_{i} v_{i}\right)\right)}{\partial \beta^{\tau}\left(u_{j} v_{j}\right)}=\sum_{j=1}^{\pi}\left(y_{j} x_{j}-x_{j} \exp \left(x_{j}^{\tau} \beta\left(u_{j} v_{j}\right)\right)\right) w_{j}\left(u_{j} v_{j}\right)=0
$$

The above equation is an implicit equation, so that to finish it, it is used a numerical iterative procedure i.e. NewtonRaphson method $[9,10]$. In general, the equation for NewtonRaphson iteration is as follows:

$$
\beta_{(m+1)}\left(u v_{i}\right)=\beta_{m}\left(u_{i} v_{i}\right)-H_{m}^{-1}\left(\beta_{m}\left(u_{i} v_{i}\right)\right) g_{(m)}\left(\beta_{m}\left(u_{i} v_{i}\right)\right)(7)
$$

Where

$$
\begin{gathered}
\boldsymbol{g}_{(m)}\left(\beta_{m}\left(u_{i}, v_{i}\right)\right)=\frac{\partial \operatorname{Ln} L^{*}\left(\beta\left(u_{i}, v_{i}\right)\right)}{\partial \beta^{T}\left(u_{i}, v_{i}\right)} \\
=\sum_{i=1}^{n} x_{i} w_{i j}\left(u_{j}, v_{j}\right)-\sum_{i=1}^{n} x_{i} w_{i j}\left(u_{j}, v_{j}\right) \exp \left(x_{i}^{\tau}, \beta\left(u_{i}, v_{i}\right)\right) \\
=\sum_{i=1}^{n}\left[x_{j} w_{i j}\left(u_{j} v_{j}\right)-x_{j} w_{i j}\left(u_{j} v_{j}\right) \exp \left(x_{i}^{T}, \beta\left(u_{i} v_{i}\right)\right)\right](8) \\
=-\sum_{i=1}^{n} x_{i} w_{i j}\left(u_{j}, v_{j}\right) \exp \left(x_{i}^{T}, \beta\left(u_{i}, v_{i}\right)\right)+\sum_{i=1}^{n} x_{i} w_{i j}\left(u_{j}, v_{j}\right) \\
H_{m}^{-1}\left(\beta_{(m)}\left(u_{i} v_{i}\right)\right)=\frac{\partial \operatorname{Ln} L^{2}\left(\beta\left(u_{i} v_{i}\right)\right)}{\partial \beta\left(u_{i} v_{i}\right) \partial \beta^{T}\left(u_{i} v_{i}\right)} \\
=-\sum_{i=1}^{n} x_{i} w_{i j}\left(u_{i} v_{i}\right) x_{i}^{T} \exp \left(x_{i}^{T} \beta\left(u_{i} v_{i}\right)\right)
\end{gathered}
$$

If equation (8) and (9) are substituted into equation (7), it is obtained:

$$
\begin{aligned}
& \hat{\beta}_{(m+1)}\left(u_{i} v_{i}\right)=\beta_{(m)}\left(u_{i} v_{i}\right)-H_{m}^{-1}\left(\beta_{(m)}\left(u_{i} v_{i}\right)\right) g_{(m)}\left(\beta_{(m)}\left(u_{i} v_{i}\right)\right) \\
& \beta_{m+1)}\left(u_{i} v_{i}\right)=\left(X^{\tau} W\left(u_{i} v_{i}\right) A\left(u_{i} v_{i}\right)^{(m)} X\right)^{-1}\left(X^{\tau} W\left(u_{i} v_{l}\right) A\left(u_{i} v_{l}\right)^{(m)} z\left(u_{i} v_{i}\right)^{(m)}\right)
\end{aligned}
$$

If matrix approach is used, equation (10) can be written as follows:

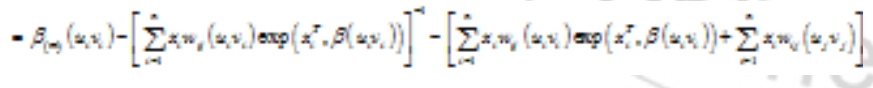

Where

$\mathrm{X}$ : predictor matrix, is denoted as follows:

$$
X=\left[\begin{array}{ccrll}
1 & x_{11} & x_{12} & \ldots & x_{1 p} \\
1 & x_{21} & x_{22} & \ldots & x_{2 p} \\
\vdots & \vdots & \vdots & \ddots & \vdots \\
1 & x_{31} & x_{32} & \ldots & x_{3 p}
\end{array}\right]
$$

$W\left(u_{i}, v_{i}\right)$ : weighting matrix, is denoted as follows: $W\left(u_{i}, v_{i}\right)=\operatorname{diag}\left[\begin{array}{llll}w_{i 1} & w_{i 2} & \ldots & w_{i n}\end{array}\right]$

$W\left(u_{i}, v_{i}\right)$ : Variant weighting matrix for each location of $i$ $W\left(u_{i}, v_{i}\right)$ : Vector of response variables, defined as follows:

$$
z^{(m)}\left(u_{i}, v_{i}\right)=\left(z_{1}^{(m)}\left(u_{i}, v_{i}\right), z_{2}^{(m)}\left(u_{i}, v_{i}\right), \ldots, z_{n}^{(m)}\left(u_{i}, v_{i}\right)\right)
$$

By repeating iterative procedure for each regression point $i$ order, local parameter estimator will be obtained. Iteration stops in convergent time that is when $\left\|\beta^{(m+1)}\left(u_{i}, v_{i}\right)-\beta^{(m)}\left(u_{i}, v_{i}\right)\right\| \leq \varepsilon$, where $\varepsilon$ is a very small number.

The first hypothesis test performed is the similarity test of Poisson regression model and GWPR model to test the significance of geographical factors. The form of the hypothesis is:

$H_{0}: \beta_{k}\left(u_{i}, v_{i}\right)=\beta_{k} \quad ; i=1,2, \ldots, n ; k=1,2, \ldots, p$

(There is no significant difference between Poisson regression model and GWPR model)

$H_{1}: \beta_{k}\left(u_{i}, v_{i}\right) \neq \beta_{k}$ (There is significant difference between Poisson regression model and GWPR model).

The similarity test of Poisson regression model and GWPR model use deviant value comparison of Poisson regression model and GWPR model. If Poisson regression model is expressed by model $\mathrm{A}$ with degree of freedom $d f_{A}$ and GWPR model is expressed by model $B$ with degree of freedom $d f_{B}$ so that:

$$
F_{\text {test }}=\frac{\text { Devians Model } A / d f_{A}}{\text { Devians Model } B / d f_{B}}
$$

Will follow the distribution of $F$ with degree of freedom $d f_{A}$ and $d f_{B}$. The testing criteria is reject $H_{0}$ if

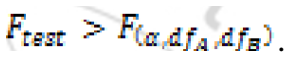

Hypothesis for testing the significance for the local version of the $k^{\text {th }}$ parameter estimate is described as:

$$
\begin{aligned}
& H_{0}: \beta_{k}\left(u_{i}, v_{i}\right)=0 \\
& H_{1}: \exists_{k} \text { where } \beta_{k}\left(u_{i}, v_{i}\right) \neq 0 ; k=0,1,2, \ldots,(p-1)
\end{aligned}
$$

The local pseudo t-statistic for the local version of the $k^{\text {th }}$ parameter estimate is then computed by:

$$
t_{k}\left(u_{i}, v_{i}\right)=\frac{\hat{\beta}_{k}\left(u_{i}, v_{i}\right)}{\operatorname{Se}\left(\hat{\beta}_{k}\left(u_{i}, v_{i}\right)\right)}
$$

This can be used for local inspection of parameter significance[7].

The standard error of $\hat{\beta}_{k}\left(u_{i}, v_{i}\right)$, the estimate of the $k^{\text {th }}$ regression coefficient at the $i^{\text {th }}$ data point, is given by:

$$
\operatorname{Se}\left(\beta_{k}\left(u_{i}, v_{i}\right)\right)=\sqrt{\operatorname{cov}\left(\hat{\beta}_{k}\left(u_{i}, v_{i}\right)\right)}
$$

With $\operatorname{vor}\left(\hat{\beta}_{k}\left(u_{1}, v_{1}\right)\right)$ as the diagonal $k$-order on matrix $\operatorname{var}\left(\hat{\beta}\left(u_{i}, v_{i}\right)\right)$ having size of $((p+1) x(p+1))$ and $\hat{\beta}_{k}\left(u_{i}, v_{i}\right)$ which is the model parameter estimation maximizing log-likelihood function. The usual threshold of $p$-values for a significance test is effectively $|t|>1.64$ for tests at the ten percent level with large samples[10].

The weighting used to estimate the parameters in GWPR model is kernel function consisted of Gaussian kernel function and Bisquare kernel function which can be written as follows:

1. Gaussian Kernel Function

$$
w_{j}\left(u_{i}, v_{i}\right)=\exp \left[-\frac{1}{2}\left(\frac{d_{i j}}{h}\right)^{2}\right]
$$

2. Bisquare Kernel 


\section{International Journal of Science and Research (IJSR) \\ ISSN (Online): 2319-7064}

Index Copernicus Value (2013): 6.14 | Impact Factor (2014): 5.611

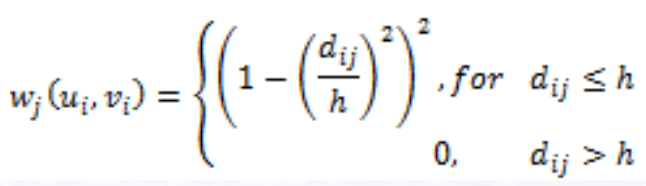

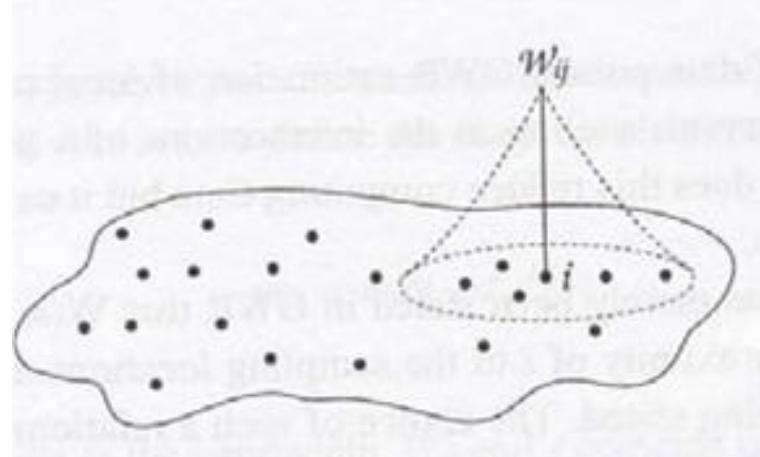

Figure 1: Schematic representation of the kernel' bandwith and its shape

With $d_{i j}=\sqrt{\left(u_{i}-u_{j}\right)^{2}+\left(v_{i}-v_{j}\right)^{2}}$,

between locations $\left(u_{i}, v_{i}\right)$ to location $\left(u_{j}, v_{j}\right)$ and $h$ is nonnegative parameter usually known and called as smoothing parameter or bandwidth[1]. If the weight used is kernel function, then the choice of bandwidth is very important because bandwidth is a balance controller between curves towards data and data smoothness [1,4,6,7]. The method used to choose optimum bandwidth is Cross Validation (CV). This method is noted as below:

$C V(h)=\sum_{i=1}^{n}\left(y_{i}(h)-\hat{y}_{\neq i}(h)\right)^{2}$

with:

$y_{i}(h)$ : fitting value $y_{i}$ in which observation on location $\left(u_{i}, v_{i}\right)$ is omitted from estimation process.

$\hat{y}_{\neq i}(h)$ : fitting value $y_{i}$ in which observation on location $\left(u_{i}, v_{i}\right)$ is included in the estimation process.

n $\quad$ : sample total number.

Omitting this data point is necessary since minimizing the above equation using all observations results in the fitted values tending toward their corresponding actual values (i.e., the $C V$ score tends toward zero). This occurs when the weights for all other data points (except for the $i^{\text {th }}$ data point) tend to zero, which occurs when the value of $h, d$ or $M$ tends to zero. The $C V$ or 'leave-one-out' score can be regarded as the sum of' the squared errors associated with estimating $\hat{y}_{\neq i}(h)$ at each data point, where each point contributes toward the total $C V$ score $[9,10]$.

The method used to select the best model for GWPR is Akaike Information Criterion (AIC) which is defined as follows:

$$
A I C=D(G)+2 K(G)
$$

with $D(G)$ is the model deviant value with bandwidth $(G)$ and $K(G)$ is the number of parameters in the model with bandwidth $(G)$. In addition to GWPR, the AIC can also be used for choosing a suitable value of $\mathrm{h}, \mathrm{d}$ or $\mathrm{M}$ in logistic and other geographically weighted generalized linear models [7].
The best model is the model with the smallest $A I C$ value, then there is evidence to suggest that the relationship between the response variable and the predictor variable of interest varies spatially, there is little evidence to suggest that the relationship varies over space.

\section{Data Analysis and Discussion}

\subsection{Title and authors}

As a starting point for GWPR models analysis, it was necessary to set up global regression namely Poisson regression model. Before forming Poisson regression it needed to have collinearity test to determine whether the predictor variables had fulfilled the conditions which were not mutually correlated.

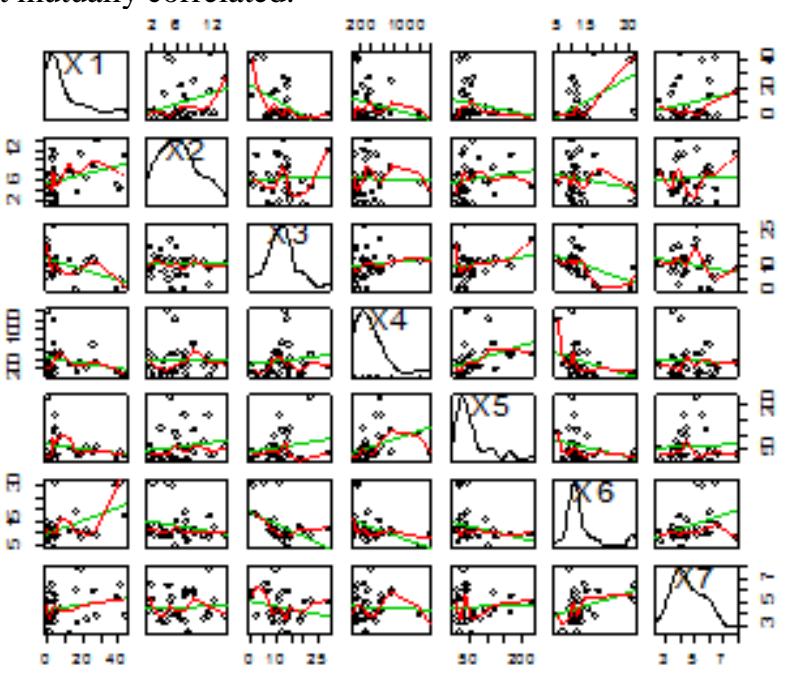

Figure 2: Scatterplot Correlation Matrix of Variables

Several criteria could be used to determine the presence of collinearity among predictor variables by using coefficient correlation (Pearson Correlation) and the value of Variance Inflation Factor (VIF). Both criteria showed similar result i.e. there was no collinearity among predictor variables so that the predictor variables used in this study in North Sumatra Province in 2013 could be used in the formation of Poisson Regression Model. The following table shows the Poisson regression model parameter estimation in North Sumatera Province.

Table 1: Poisson Regression Model Parameter Estimation

\begin{tabular}{|c|c|c|c|c|}
\hline Parameter & Estimate & Standard Error & $Z($ Est $/ S E)$ & $\operatorname{Pr}(>|z|)$ \\
\hline$\beta_{0}$ & 1.59993 & 0.07892 & 20.27192 & 0.00953 \\
\hline$\beta_{1}$ & -0.14603 & 0.10106 & -1.44498 & 0.14836 \\
\hline$\beta_{2}$ & 0.08544 & 0.09399 & 0.90907 & 0.36321 \\
\hline$\beta_{3}$ & 0.01712 & 0.08891 & 0.19258 & 0.84718 \\
\hline$\beta_{4}$ & 0.08414 & 0.08412 & 1.00028 & 0.31704 \\
\hline$\beta_{5}$ & -0.09321 & 0.09212 & -0.19301 & 0.31160 \\
\hline$\beta_{6}$ & 0.19615 & 0.20888 & 1.80161 & 0.07155 \\
\hline$\beta_{7}$ & -0.04158 & 0.08850 & -0.46978 & 0.63859 \\
\hline
\end{tabular}

From Table 1 it can be seen that with level of significance of $10 \% \quad\left(\mathrm{Z}_{0.05}=1.645\right)$ it was obtained two significant parameters having influence namely $\beta_{0}$ and $\beta_{6}$. Thus, 


\section{International Journal of Science and Research (IJSR) \\ ISSN (Online): 2319-7064 \\ Index Copernicus Value (2013): 6.14 | Impact Factor (2014): 5.611}

Poisson regression model formed for the percentage of infant mortality in North Sumatera Province was:

$\mu_{i}=\exp \left(1.59993+0.19615 X_{6}\right)$

The model above explained that the percentage of infant mortality would increase by $\exp (0.19615)$ if poor variable increased by one unit with condition if other predictor variables were constant. The same interpretation applied to other predictor variables.

The first analysis step in GWPR is determining the bandwith used in Gaussian Kernel weight function. The optimum bandwith selection is a feature of the golden section search method in the GWR4 software[6]. In selecting the optimum bandwith, GWPR model with Fixed Gaussian Kernel weighting function has an AIC value of 91.003 and with Fixed bi-square has an AIC value of 62.274.

Table 2: Summary Statistics of GWPR parameter estimates : Fixed Gaussian Weighting Function with $h=0.911$

\begin{tabular}{|c|c|c|c|}
\hline Covariate & Mean & Robust STD & IQR \\
\hline Intercept & 1.453 & 0.104 & 0.140 \\
\hline$X_{1}$ & -0.235 & 0.131 & 0.177 \\
\hline$X_{2}$ & 0.105 & 0.023 & 0.031 \\
\hline$X_{3}$ & 0.006 & 0.102 & 0.138 \\
\hline$X_{4}$ & 0.076 & 0.037 & 0.050 \\
\hline$X_{5}$ & -0.105 & 0.059 & 0.079 \\
\hline$X_{6}$ & 0.090 & 0.181 & 0.244 \\
\hline$X_{7}$ & -0.018 & 0.034 & 0.046 \\
\hline AIC & 91.003411 & \\
\hline
\end{tabular}

Table 3: Summary Statistics of GWPR parameter estimates: Fixed Bi-square Weighting Function with $\boldsymbol{h}=\mathbf{2}, \mathbf{8 5 8}$

\begin{tabular}{|c|c|c|c|}
\hline & Mean & Robust STD & IQR \\
\hline Intercept & 1.478 & 0.054 & 0.072 \\
\hline$X_{1}$ & -0.212 & 0.079 & 0.106 \\
\hline$X_{2}$ & 0.106 & 0.016 & 0.021 \\
\hline$X_{3}$ & 0.007 & 0.094 & 0.127 \\
\hline$X_{4}$ & 0.081 & 0.023 & 0.031 \\
\hline$X_{5}$ & -0.104 & 0.028 & 0.038 \\
\hline$X_{6}$ & 0.113 & 0.140 & 0.189 \\
\hline$X_{7}$ & -0.020 & 0.018 & 0.024 \\
\hline AIC & \multicolumn{3}{|c}{62.373522} \\
\hline
\end{tabular}

Based on Table 2 to 3 show that GWPR model with Fixed $\mathrm{Bi}$-square weighting function was better in use for analyzing the number of infant mortality in North Sumatera province because it had the smallest AIC value.

\section{Conclusions and Recommendation}

From the data analysis and discussion, it can be concluded as follows:

1. GWPR model with Fixed Bi-square weighting function performed better and provide significant improvement over the fixed Gaussian kernel.

2. The local t-test did not perform well for the single predictor case, with the power of the test being very low (less then 10\%) for all calibrated GWPR models. However, the power improved slightly when the value of thee weighting function parameter decreased.

However, further studies may be needed to examine the performance of a mixed GWPR model, with respect to the degree of bias in the regression coefficients, selection of the weighting function parameter which yields the lowest AIC value and $\mathrm{CV}$ score, as well as hypothesis testing for the significance of the regression coefficients and the power of these test.

\section{References}

[1] A.S. Fotheringham, Brundson, C., and Charlton, M. "Geographically Weighted Regression : The Analysis of Spatially Varying Relationships," Chichester: John Wiley. 2002

[2] BPS, "Sumatera Utara In Figures 2014," Indonesia, Jakarta. 2014.

[3] BPS, "Angka Kematian Bayi, Data Statistik Indonesia," Indonesia, Jakarta. 2009

[4] C.L. Mei, "Geographically Weighted Regression Technique for Spatial Data Analysis," School of Science Xi'an Jiaotong University. 2005.

[5] N. Draper and H. Smith, "Applied Regression Analysis Third Edition," New York, Wiley. 1998.

[6] N. Tomoki, M. Charlton, P. Lewis, C. Brundson, J. Yao and Fotheringham, A.S., "GWR4 User Manual: Windows Application for Geographically Weighted Regression Modelling," Ritsumeikan University : Departement of Geography. 2014.

[7] M.C. Sean, "An Application of Geographically Weighted poisson Regression," Newfoundland, Canada. 2010

[8] M. Charlton, and A.S. Fotheringham "Geographically Weighted Regression," White paper, Maynooth, Co Kildare, Ireland. 2009.

[9] S. Farber \& A. Paez "A Systematic Investigation of Cross-Validation in GWR Model Estimation: Empirical Analysis of Spatial Non-Stationarity," Journal of Geographical Systems, 9, 371-396.

[10] T. Nayaka, Fotheringham AS, Brundson C, and Charlton M., "Geographically Weighted Poisson Regression for disease Association Mapping," Statistics in Medicine, 24: 2695-2717. 2005) variable have not significant impact 